\title{
Device Size Text
}

National Cancer Institute

\section{Source}

National Cancer Institute. Device Size Text. NCI Thesaurus. Code C106041.

Information about the size of the device that is not represented elsewhere. 